\title{
G schestem \\ Design and Implementation of 16 Quality Indices for Neonatal Screening
}

\section{Yao Chen}

Fujian Provincial Maternity and Children's Hospital: Fujian Provincial Maternity and Children's Hospital

\section{Yinglin Zeng}

Fujian Provincial Maternity and Children's Hospital: Fujian Provincial Maternity and Children's Hospital

\section{Xiaolong Qiu}

Fujian Provincial Maternity and Children's Hospital: Fujian Provincial Maternity and Children's Hospital

\section{Feng Lin}

Fujian Provincial Maternity and Children's Hospital: Fujian Provincial Maternity and Children's Hospital

\section{Hong Zhao}

Fujian Provincial Maternity and Children's Hospital: Fujian Provincial Maternity and Children's Hospital Honghua Zhang

Fujian Provincial Maternity and Children's Hospital: Fujian Provincial Maternity and Children's Hospital

\section{Weifen Chen}

Fujian Provincial Maternity and Children's Hospital: Fujian Provincial Maternity and Children's Hospital

\section{Qingying Lin}

Fujian Provincial Maternity and Children's Hospital: Fujian Provincial Maternity and Children's Hospital

\section{Guanghua Liu}

Fujian Provincial Maternity and Children's Hospital: Fujian Provincial Maternity and Children's Hospital

\section{Wenbin Zhu ( $\square$ fjnsczwb@fjmu.edu.cn )}

Fujian Provincial Maternity and Children's Hospital: Fujian Provincial Maternity and Children's Hospital https://orcid.org/0000-0001-7439-7504

\section{Research}

Keywords: Neonatal Screening, 16 quality indicators, Quality Index, Evaluate, dynamic management

Posted Date: May 25th, 2021

DOl: https://doi.org/10.21203/rs.3.rs-520651/v1

License: (9) (1) This work is licensed under a Creative Commons Attribution 4.0 International License. Read Full License 


\section{Abstract}

Background: We aim to explore the realization of 16 quality control indices of neonatal disease screening in neonatal disease management information system, and to establish the multi-center management evaluation model under the framework of the whole province.

Methods: Based on the network management system for neonatal screening originally used in our province, the quality management module of neonatal disease was developed and applied according to the quality control index. A quantitative evaluation method was established for the quality of all maternal and child health care institutions involved in neonatal disease screening related to neonatal disease screening, specimen collection and recall of suspected cases.

Results: The quality management information was shared by all neonatal screening agencies. Comprehensive evaluation was given to the multi-center quality management measurements. Comprehensive evaluation was performed to quality among different medical institutions. Evaluation of applicability and timeliness of quality in practical work was conducted accordingly.

Conclusions: The timeliness of self-improvement in centers and medical institutions were improved. The application of neonatal disease screening quality management index improved the requirements for neonatal screening management. The quality management module was adopted to conduct dynamic management of the whole management process, which greatly improved the overall management level of neonatal screening.

\section{Background}

Neonatal screening, with the aim to detect newborns with serious, treatable disorders so as to facilitate appropriate interventions, involves the cooperation of medical institutions from each location. On this basis, it is necessary to establish single or multiple screening centers that consist of a huge network for the screening, under the guidance and supervision of the corresponding authorities. With the advances of neonatal screening, the screening volume is on an increasing trend, which extends to the rural areas reaching millions of individuals or even more. In order to guarantee the whole screening efficiency, a complete management system is required, which is backed up by modern computer information management system [1-5].

In China mainland, the neonatal screening system, proposed by the provincial medical authorities, is a public medical program involving the establishment of screening management network and conduction of screening by institutions. Its management is an evaluation item for the local authorities, and the priority is of prime importance. Thus, extensive efforts have been reported on the establishment of screening quality and evaluation, standardization of screening progress, as well as improving the service quality. Neonatal screening diagnosis is highly depending on the recall of aberrant screening findings in the near future. In cases of any misdiagnosis or missing of any aberrant findings, it may trigger disastrous conditions afterwards. On this basis, it is crucial to manage the screening quality and control, 
in order to guarantee the screening quality and safety. In China, since 1998, extensive studies have been focusing on the quality evaluation of screening laboratories [6, 7], according to the Newborn Screening Technical assistance and Evaluation Program (NewSTEPs). This lay the foundation for the quality evaluation program for the genetic metabolism screening in the neonates in China, which is featured by investigation of corresponding indices in a provincial state, together with the quality evaluation program for clinical laboratory center by the Ministry of Health and the quality evaluation activity for the genetic metabolism disease screening in the neonates [7-9]. In 2017, the consensus for the quality indicators of neonatal screening for inherited metabolic disease issued by National Health Commission of the PRC proposed 16 evaluation indices for the neonatal screening, which pave the way for the screening evaluation system for the genetic metabolism disease in the neonates in China mainland. The screening management involves various processes including signing informed consent, sample collection and transmission, sample determination and re-examination, disease diagnosis and typing, as well as treatment and follow up [10]. The procedures are rather complex with various institutions and personnel involved, which requires standardization in detail. In this study, we aim to standardize these procedures, which can provide basis for the neonatal screening.

\section{Methods}

\section{Screening management progress}

Neonatal screening is a complex system involving organization management, laboratory technique, clinical diagnosis and public education, which requires complete cooperation of each department. This process put a harsh demanding on the scientificity and technology, as well as a standard management. New screening involves several fields, including clinical medicine, genetics, principles of management, sociology, informatics and education, and the whole process is rather complex including signing informed consent, sample collection and transmission, determination, diagnosis, treatment, follow up and evaluation, by the doctors, nurses, laboratory staff, public health doctors, statistician, quality control personnel, and social staff. This involves cooperation of many medical institutions including screening center, sample collection and quality control center, institutions for the recall of cases with positive screening, and administrative management department. The system is rather complex and timeconsuming, which involves management of massive information, data on the recall and diagnosis and follow up. To ensure whole system quality, standard management model and information management system are required, and continuous efforts are needed [1-5].

\section{Constitution of the new screening management system}

Process for the new screening included informed consent, sample collection and transmission, determination, recall, diagnosis, treatment, follow up and evaluation. Information management system (IMS) is designed and utilized based on these aspects, including the blood collection system, transmission system, laboratory, diagnosis, treatment and follow up systems that are closely related to each other (Figure 1). 


\section{Division of the screening management system based on laboratory management}

A management theory with the core of complete quality control before, during and after analysis was established. Before analysis, blood sample collection was conducted, until sample transmission and management, and sample reception. During the analysis, it started with the management of laboratory and ended with the data upload, quality control analysis and data interpretation. Quality control after analysis included report release, notice management, recall of aberrant data and follow up management (Figure 2).

\section{Evaluation of disease screening}

In order to standardize the quality management of genetic metabolic disease by neonatal screening, 16 evaluation indices were proposed according to the previous description [2], together with the separation and location of the screening management system. This process is used for quality evaluation of screening, which guarantees the progressing of screening of metabolic disease in neonates.

Division of the 16 indices was based on the function. Sample collection was mainly focused on the evaluation of health education rate, screening rate, Percentage of unqualified blood spots, percentage of important information miss, turnover time of dry blood sample, in time rate, and positive rate of prescreening. Screening laboratory was mainly used for the release time of report, in time rate, room quality control rate, rate of failure for quality control CV. Recall department was mainly responsible for the positive rate of recall and rate of lost in the follow up. Comprehensive control index included positive rate of recall, prediction of positivity, prevalence of screening disease, as well as false negativity.

In order to achieve 16 indices of quality control in neonatal screening, numeralization was used for quality control based on certain conditions, in order to obtain comparability of quality control indices. Meanwhile, normalization management was given in actual process (Figure 3). Furthermore, quality control evaluation system was established during the running of the system, which guaranteed the continuous improvement of the system.

\section{Awareness of health education of neonatal screening}

For the informed consent was signed by the parents. On this basis, the information should be entered into the medical system. Meanwhile, an option should be given in the information collection file, including screening consent, blood sample collection (Figure 4). In cases of no blood sample collection, it meant not signing the file or no health education.

\section{Screening rate}

The screening rate was analyzed based on a time point of 20 days after birth. Usually, there is no time limitation, and screening data should be included in the statistical analysis. For cases born in a certain hospital and received screening in another hospital, the screening rate was bequeathed to the former hospital (Figure 5). 


\section{Percentage of non-qualified dry blood sample}

In a certain statistical cycle, there might be changes of non-qualified dry blood sample for twice or more. In cases of a single, twice or more non-qualified dry blood sample, superposition was required (Figure 6).

\section{Percentage of missing of important information in the dry blood sample}

The important information should be based on the technological specification. Missing components or requirement of modification by the approver was considered to be insufficiency of important data (Figure 7).

\section{Turnover time and in-time rate for the dry blood sample before analysis}

The statistical cycle was defined as the median turnover time and in-time rate (Figure 8).

\section{Median release time and in-time rate}

Figure 9 summarized the release time of test report and in-time rate per month. The in-time rate was satisfactory in the Jan, Feb. and Mar. of 2017, respectively.

\section{Rate of quality control}

Fixed codes were set for the quality control. Data entered the screening management system. The figure for quality control of each screening program was recorded. The screening was given according to the schedule.

\section{Fraction defective rate of $\mathrm{CV}$ of the quality control}

Fraction defective rate of $\mathrm{CV}$ of quality control was analyzed per month. As there were quality control data in the management system, the data were subject to statistical analysis based on the preset CV quality limit.

\section{Positive rate of pre-screening}

The positive rate was analyzed by a season as shown in Figure 10. In the first season of 2017, the TSH percentage was the highest among these parameters, followed by G6PD, MSMS, 17-OHP and PHE, respectively.

\section{Positive rate of screening}

It is crucial to analyze the number of positive samples that were recalled. This may cause confusion to the screening positivity. The screening positive rate was presented by seasons as shown in Figure 11. In the first season of 2017, the highest percentage was G6PD, followed by TSH, 17-OHP, MSMS and PHE, respectively. 


\section{Positive rate for recall after prescreening}

The positive rate for recall was displayed by seasons. The recall rate was nearly $90 \%$ for G6PD, MSMS, 17-OHP, PHE and TSH in Jan. 2017 and Feb. 2017, respectively. In Mar. 2017, the recall rate for these parameters was still high except PHE with a rate of merely $40 \%$ (Figure 12 ).

\section{Positive rate for recall}

As shown in Figure 13, the recall rate for G6PD was high. However, that for MSMS, 17-OHP, PHE and TSH was comparatively lower with a rate of less than $20 \%$.

\section{Positive prediction value}

The G6PD was higher than $80 \%$ as shown in Figure 14. Nevertheless, the rates for the other parameters were less than $20 \%$, respectively.

\section{Prevalence of screened disorders}

The prevalence was subject to analysis per year. Besides, the prevalence was also displayed by percentage, or permillage.

\section{False negative rate}

The false negative rate was analyzed per year. The management system was used to screen the positive samples that were recalled. It is a case form generated in the system. There was no link between the prescreening negativity and the posterior content. On this basis, it is necessary to add such data and the generated link to the treatment model in the system, together with labeling of the negative code. The link was generated after confirmation with the supervisor.

\section{Lost of follow-up}

The lost of follow-up referred to the recall of samples that should have been recalled. The rate was calculated once per year as shown in Figure 15.

\section{Discussion}

The 16 quality indices were utilized to evaluate the performance of the screening system, and the index distribution was in the anterior, middle and posterior segments. Before analysis, the samples were collected by the medical centers, and then the centers could be evaluated using the samples. After analysis, the major works involved recall of the samples by the corresponding institutions in a region or country. Besides, the recall institutions could also be evaluated. The neonatal disease screening management system is governed by the provincial institutions including Medical Administrative Department, screening center, blood sample collection institutions or the maternal and children health care organization for the recalling management. Particularly, the recall institution management 
overcomes the limitations such as separation of each screening center. Upon systemic management, the recall of the samples was performed by a local maternal and children health care center wherever it was born. Meanwhile, the data at each center can be collected simultaneously. Dynamic management could be performed to each center, blood sample collection center and maternal and children health care institutions. As the 16 indices could be displayed in number, and statistical analysis could be conducted, followed by evaluation using $Z$ analysis method [11]. This can be utilized to evaluate the performance of the medical administrative center, screening center, blood sample collection center, and maternal and children health care institutions on the neonatal screening.

The evaluation indices for the medical institutions included health education rate, screening volume and rate, percentage of missing for crucial data, fraction defective rate, blood sample turnover time, in-time rate, submission of live birth, in-time fund recovery, consumable loss, screening positive rate, prescreening positivity recall rate and positivity recall rate. Then the system would generate the data automatically according to the time range (month, season or year), which indicated in the warning mode. On this basis, the aberrant data could be identified in time, followed by persistent medication to improve the screening quality.

Indeed, there are some limitations in this study. The neonatal screening for metabolic disorders involves various aspects for the quality management, but in this study, we only selected 16 representing indices. In the daily work, more quality indices may be established by the screening center, such as non-qualified sample recollection rate. In future, it is necessary to include the management system, which contributes to the standard of screening

\section{Conclusions}

In summary, the set of screening quality control is helpful for the monitoring of the management of each screening center, which provides neonatal screening services. The screening center should perform the quality management according to these indices. Therefore, the 16 indices contribute to the evaluation of dry blood spots collection, sample transmission and in-time release of report, in-lab quality control, evaluation of efficiency of neonatal screening management, efficiency of screening method and disease conditions, as well as recall of the samples. The screening management information system was divided according to the quality control indices per month, season or year, which promotes the monitoring and improvement of the problems encountered. In fact, each center could establish more quality indices according to their conditions, such as non-qualified sample recollection rate, in order to lead to the complete quality management for the neonatal screening of the genetic metabolic diseases.

\section{List Of Abbreviations}

NewSTEPs: Newborn Screening Technical assistance and Evaluation Program; IMS: Information management system; G6PD: 6-phosphate glucose dehydrogenase deficiency; MSMS: tandem mass spectrometry; 17-OHP: 17-hydroxyproline; PHE: phenylalanine; TSH: thyroid stimulating hormone. 


\section{Declarations}

\section{Ethics approval and consent to participate}

This study was approved by the Clinical Research Ethics Committee of Fujian Provincial Maternity and Children's Hospital, Affiliated Hospital of Fujian Medical University.

\section{Consent for publication}

All participants signed informed consent prior to their participation.

\section{Availability of data and material}

All data generated or analyzed during this study are included in this published article.

\section{Competing interests}

All authors declare that there is no conflict of interest associated with their contribution to this manuscript.

\section{Funding}

This study was supported by the Scientific Foundation of Fujian Provincial Maternity and Children's Hospital, Affiliated Hospital of Fujian Medical University (No. 2017-27).

\section{Authors' contributions}

CY wrote the manuscript; ZWB and LGH revised the manuscript; ZYL, QXL, LF and ZH did the data analysis; ZHH, CWF and LQY did the data collection. All authors reviewed the manuscript.

\section{Acknowledgments}

Not applicable.

\section{References}

1. WB Z. Design and performance of B/S neonatal screening system. Zhong Guo Fu You Bao Jian Za Zhi. 2007(22(33)):4650-3.

2. ZY Z. Current status of neonatal screening in China. Zhong Guo Er Tong BaoJian Za Zhi. 2011(19(2)):97-8, 101.

3. Han L, Wang F, Wang Y, Ye J, Qiu W, Zhang H, Gao X, Gong Z, Gu X. Analysis of genetic mutations in Chinese patients with systemic primary carnitine deficiency. Eur J Med Genet. 2014;57(10):571-5.

4. Cui Q SQ, Chen JH, et al. Application of screening management information system in the neonatal diseases in Anhui Province. Zhong Guo Nong Cun Wei Sheng Shi Ye Guan Li. 2013(33(12)):1044-6. 
5. Qiu L, Ding H, Zhang YM, Kong YY, Yang HH, Pan Y. Research and application of information technology in the neonatal screening field. Chinese Journal of Child Health Care. 2011.

6. Wang ZG, Xiao peng LI, Wu P, Hospital B. Evaluation on China National External Quality Assessment for Neonatal Screening Laboratory in 2001. China Public Health. 2002.

7. Wang W ZX, Yuan S, et al. Quality control evaluation of genetic metabolism disease screening in neonates. Zhong Guo Er Tong Bao Jian Za Zhi. 2017(25(11)):1182-5.

8. Fei Y WW, Zhao HJ. Evaluation of genetic metabolism screening in Chinese neonates. Zhong Hua Jian Yan Yi Xue Za Zhi. 2016(39(7)):556-8.

9. Wang H, Wang AH, Gressner OA, Fang M, Gu X, Ji Q, Cheng SQ, Shen F, Gao CF. Association between HBV Pre-S mutations and the intracellular HBV DNAs in HBsAg-positive hepatocellular carcinoma in China. Clin Exp Med. 2015;15(4):483-91.

10. The expert group of neonatal genetic screening laboratory NCfCL. Neonatal genetic and metabolic screening quality index evaluation. Zhong Hua Jian Yan Yi Xue Za Zhi. 2017(40(5)):352-5.

11. Wang J, Zhu WB, Shu YQ. APPLICATION OF Z-SCORE COMPREHENSIVE EVALUATION METHOD IN THE EVALUATION OF THE HOSPITAL NEWBORN SCREENING. Modern Preventive Medicine. 2010;99:94.

\section{Figures}

\section{Screening center}

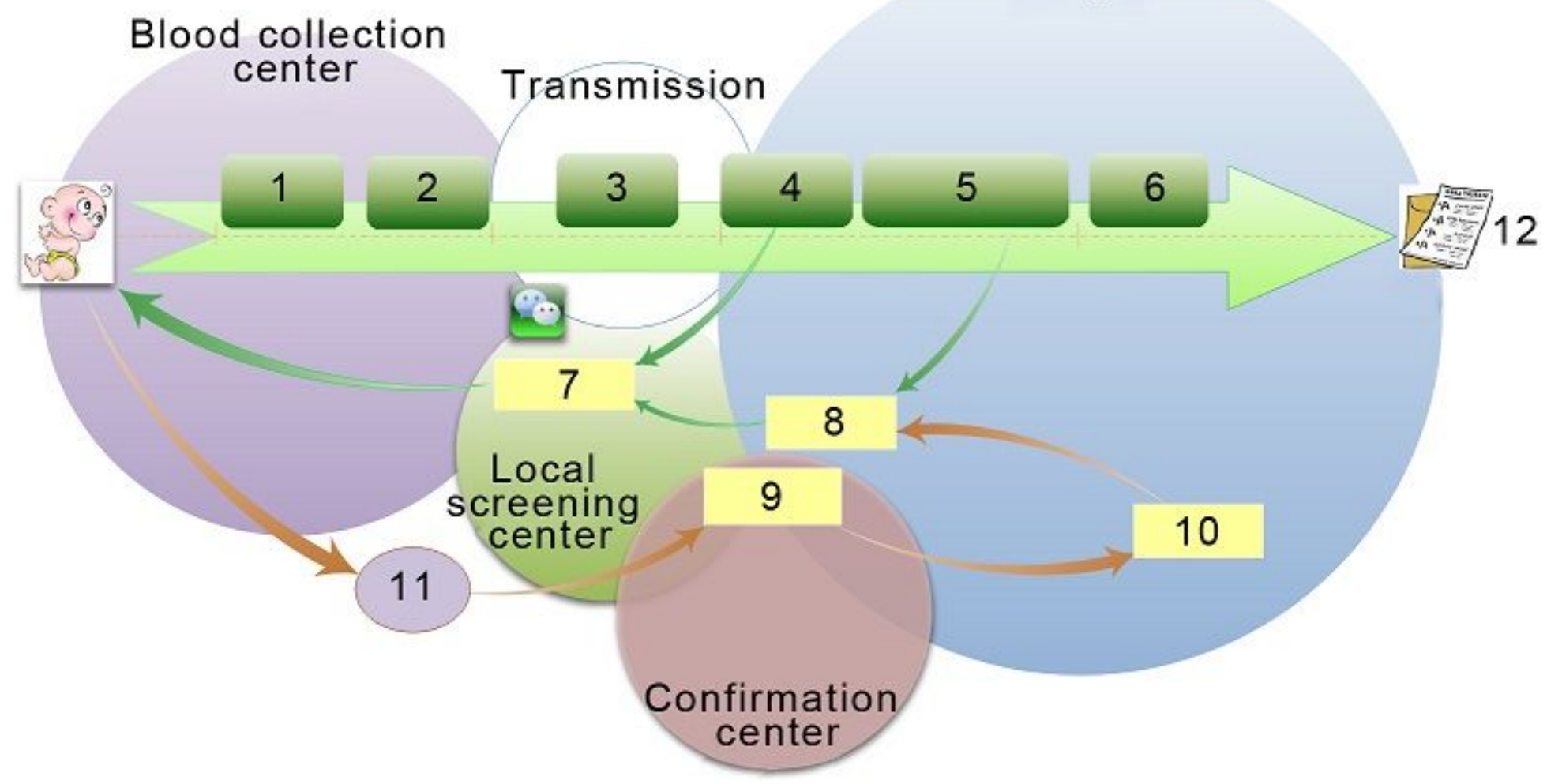

Figure 1 
Workflow. 1: Sample collection; 2: Transmission; 3: Turnover; 4: Verification; 5: Lab test; 6: Storage; 7: Recall; 8: Mal-management; 9: Confirmation; 10: Clinical diagnosis; 11: Recall and confirmation; 12 : Record center.

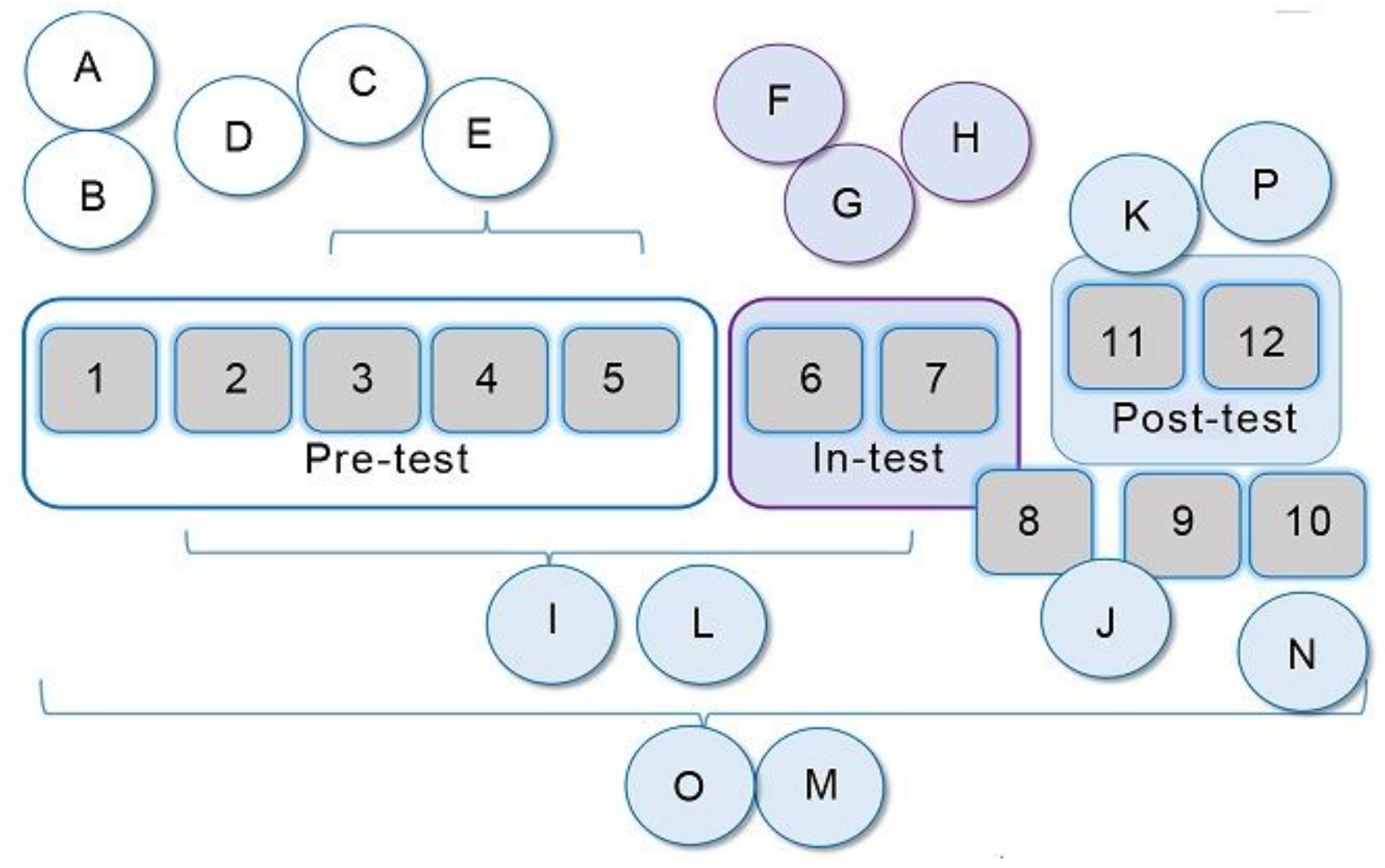

\section{Figure 2}

Element of lab quality index in the screening process. A: education rate; $\mathrm{B}$ : screening rate; C: non-qualified dry blood sample percentage; D: missing of important information; E: dry blood sample turnover and intime rate; $\mathrm{F}$ : time of report release and in-time rate; $\mathrm{G}$ : in-house quality control; $\mathrm{H}$ : non-qualified control rate; I: prescreening positivity; J: screening positivity; K: recall rate of screening positivity; L: recall positivity; M: positivity prediction; $\mathrm{N}$ : disease prevalence; $\mathrm{O}$ : prescreening negativity; $\mathrm{P}$ : lost of follow up. 1 : Education; 2 Card-filling; 3 Sample collection; 4 Transmission; 5 Recipient; 6 Test; 7 Report; 8 Prescreening; 9 Re-screening; 10 Confirmation; 11 Recall; 12 Follow up

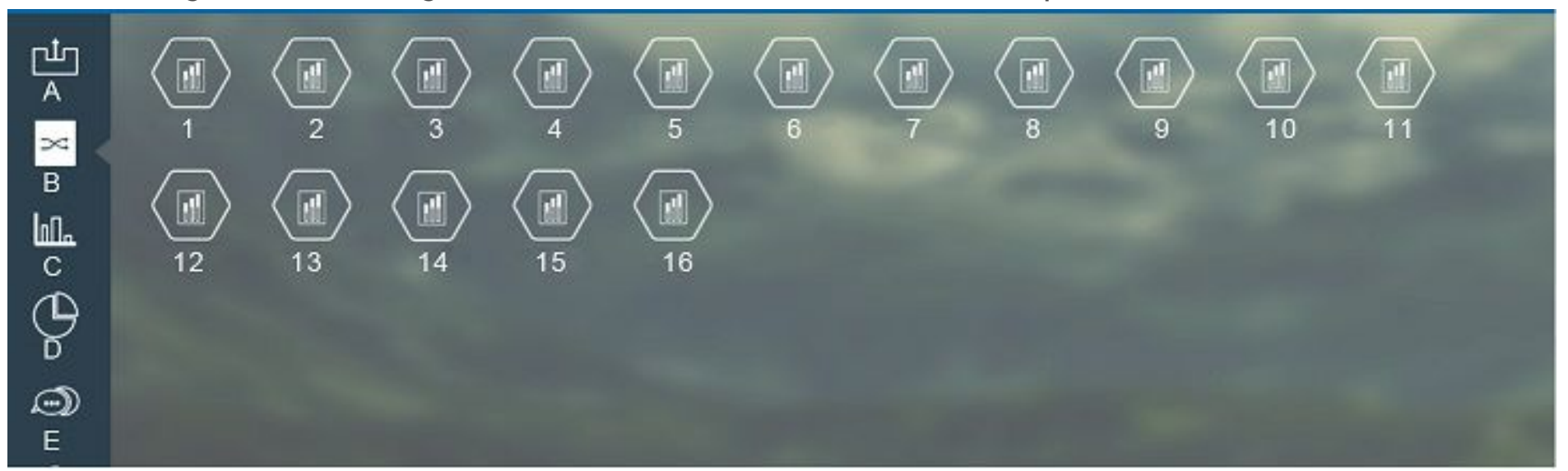




\section{Figure 3}

Summary of quality indices. A: data collection; B: in-analysis; C: in-house quality control; D: data analysis; $\mathrm{E}$ : information management. 1-16: analysis of informed rate; screening rate analysis; non-qualification analysis; data missing; dry blood sample turnover; report release; in-house quality control rate; nonqualified $\mathrm{CV}$ rate; prescreening positivity; screening positivity; recall from prescreening; recall positivity; positivity prediction; prevalence; false positivity; lost of follow up.

Percentage (\%)

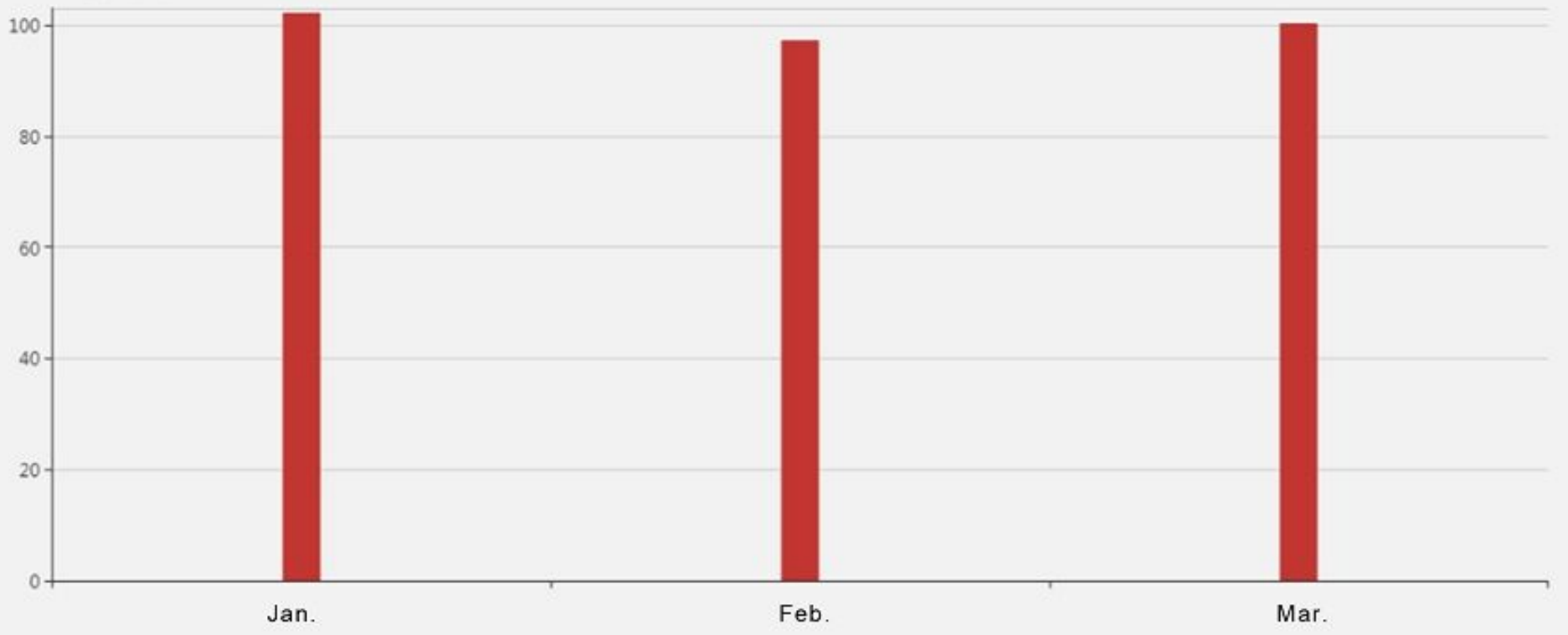

Figure 4

Informed rate of health education.

Percentage (\%)

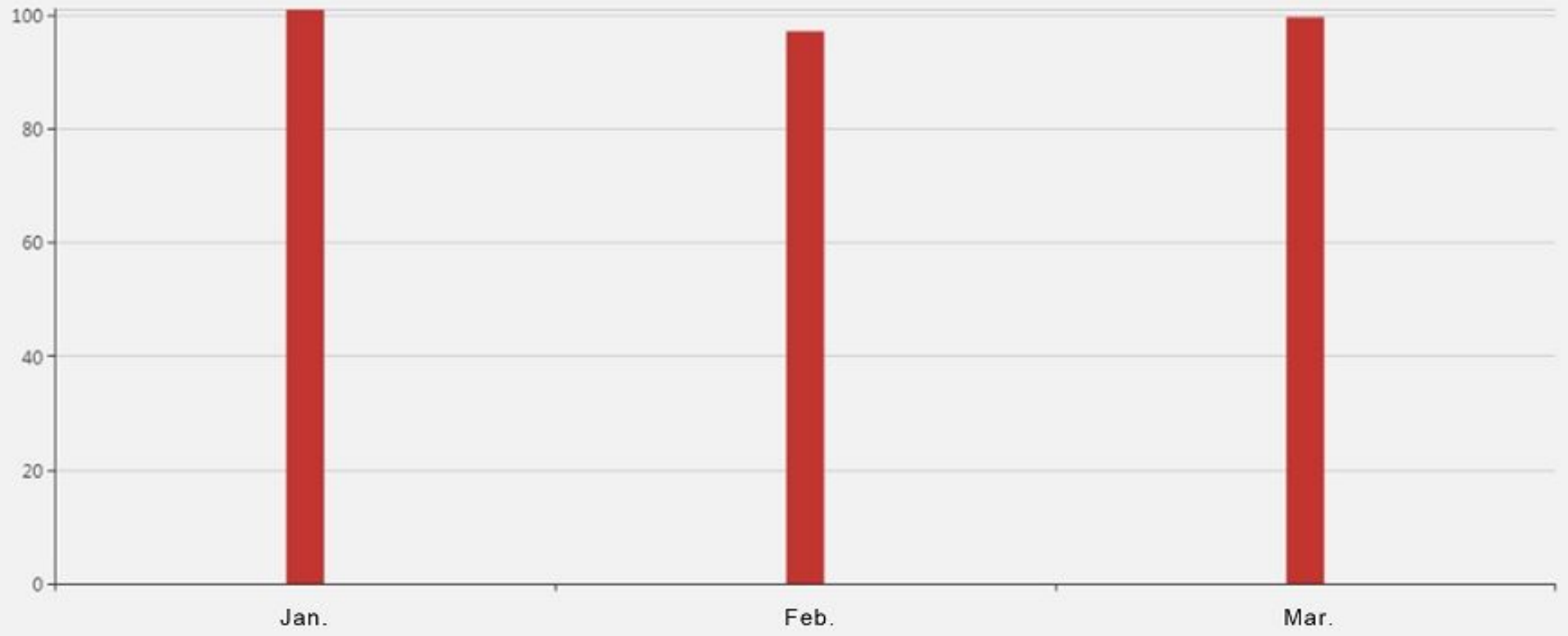

Figure 5 
Screening rate.

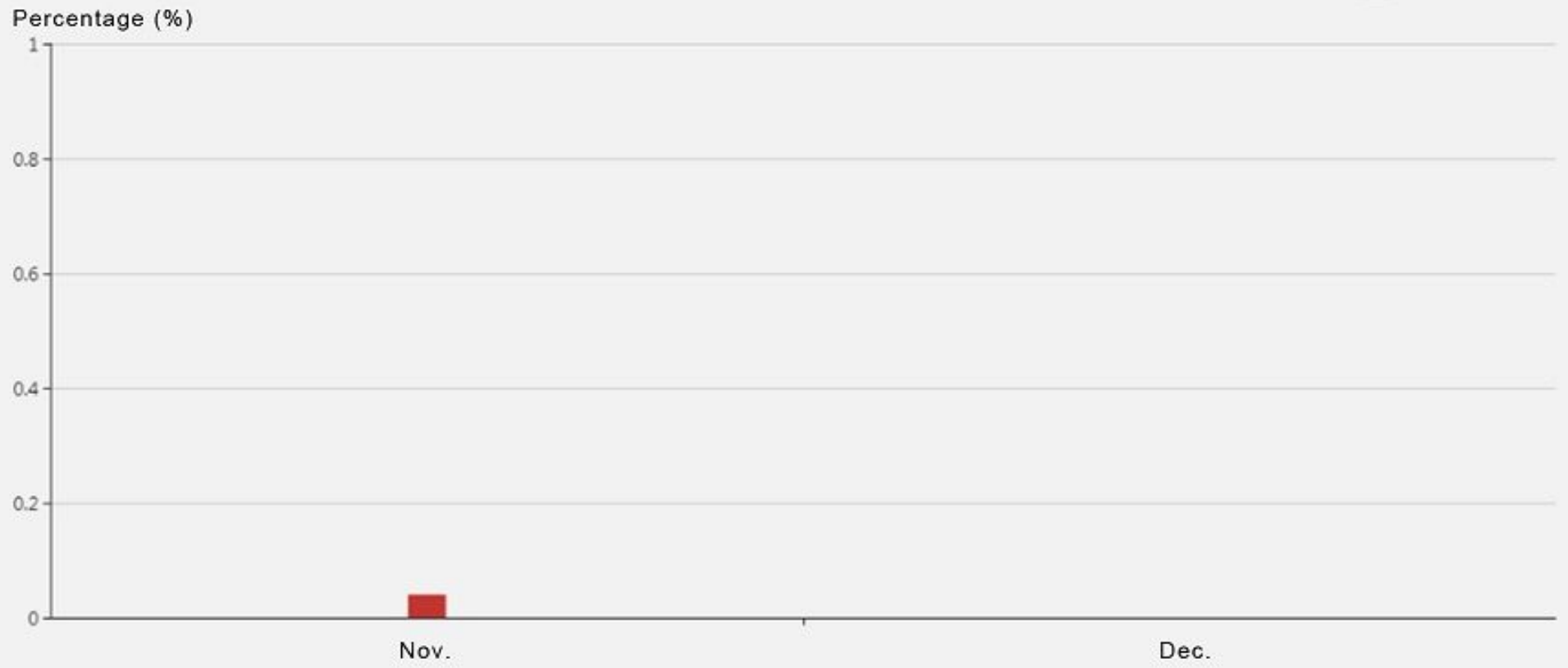

\section{Figure 6}

Percentage of non-qualified dry blood sample.

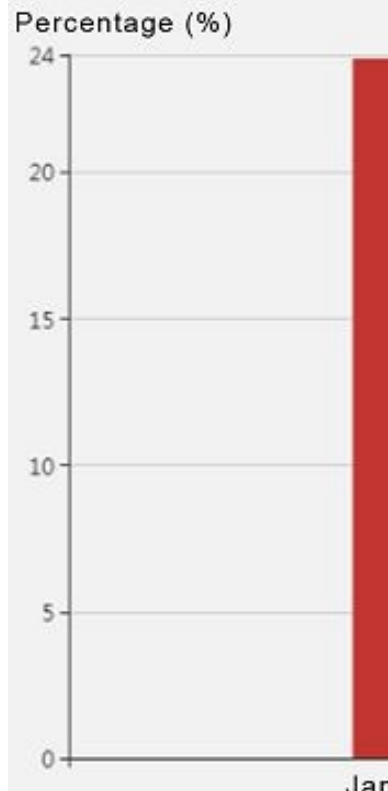

Figure 7

Percentage of missing of important information. 


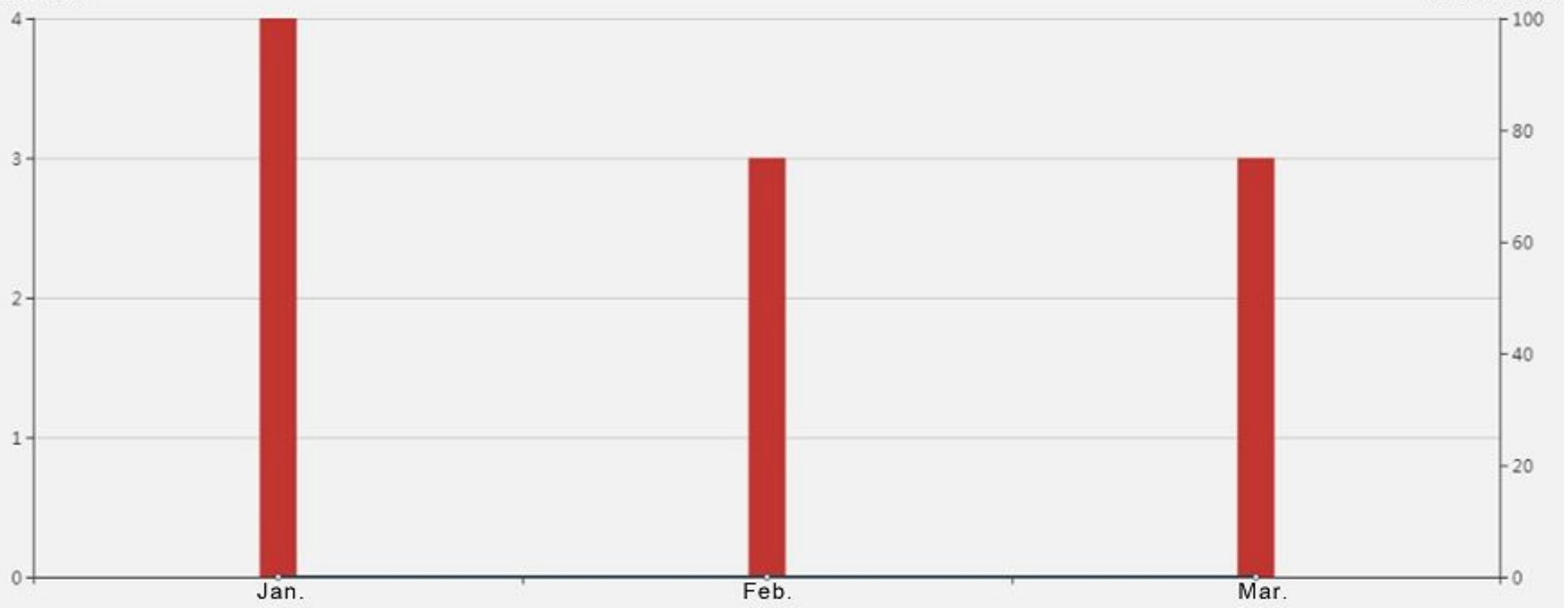

\section{Figure 8}

Median dry blood sample turnover and in-time rate before tests.

\section{Median}

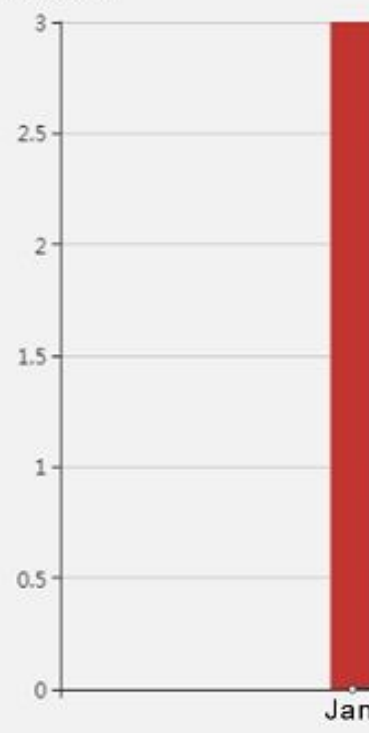

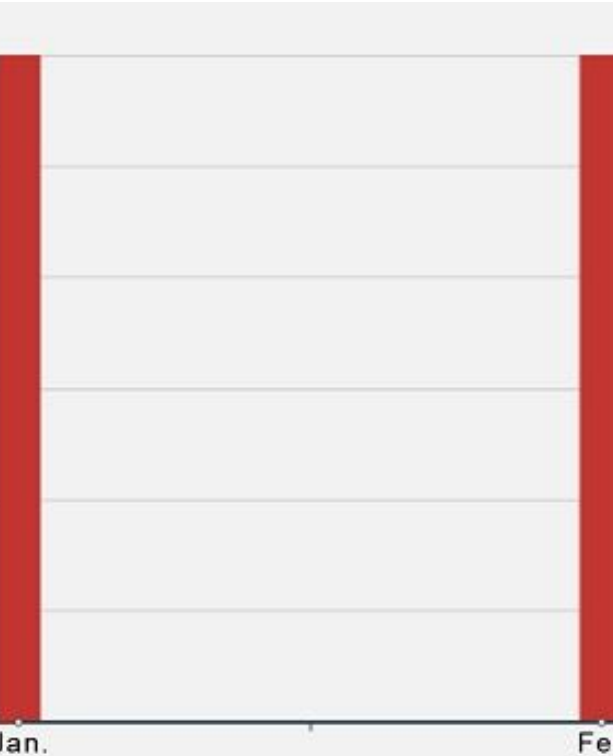

Feb.
In-time rate

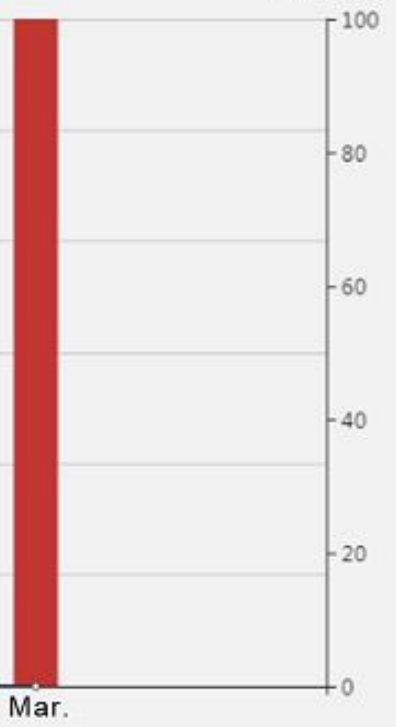

Figure 9

Median report release time and in-time rate. 


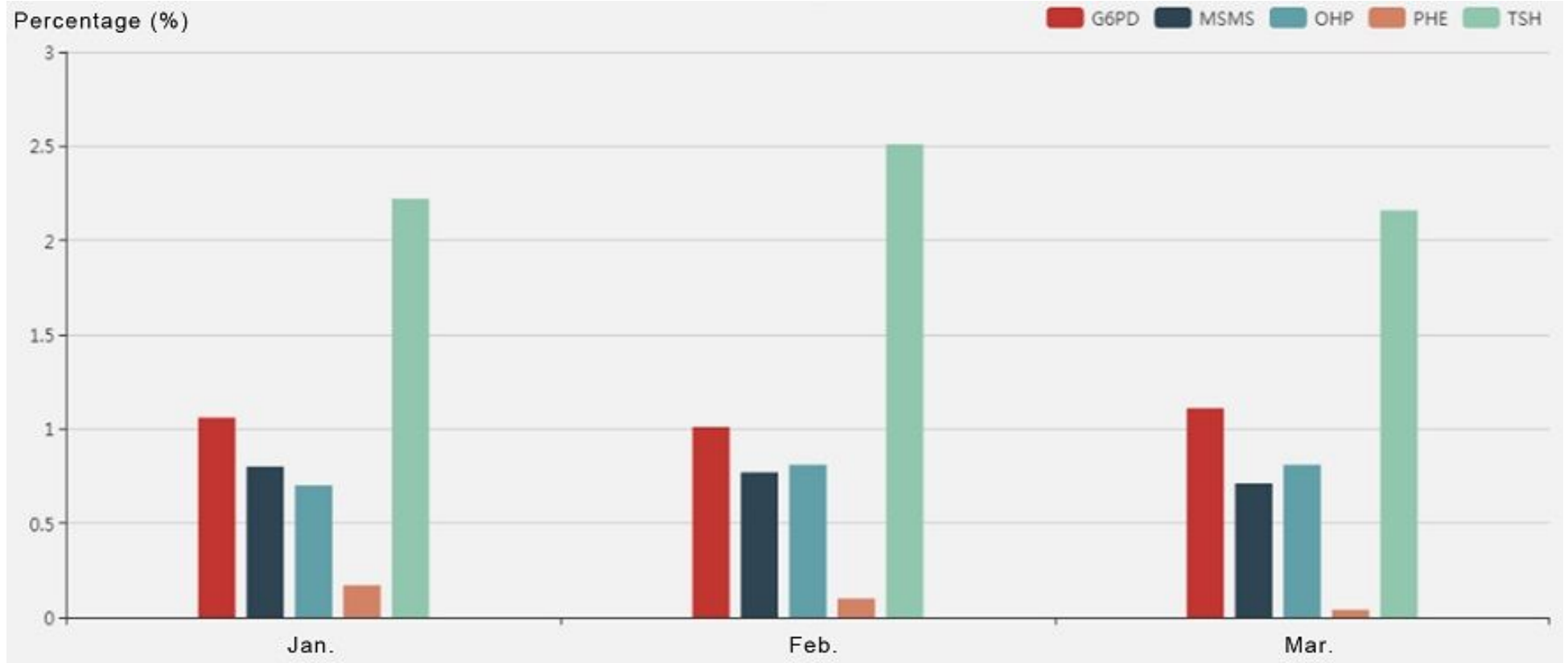

Figure 10

Positivity of pre-screening.

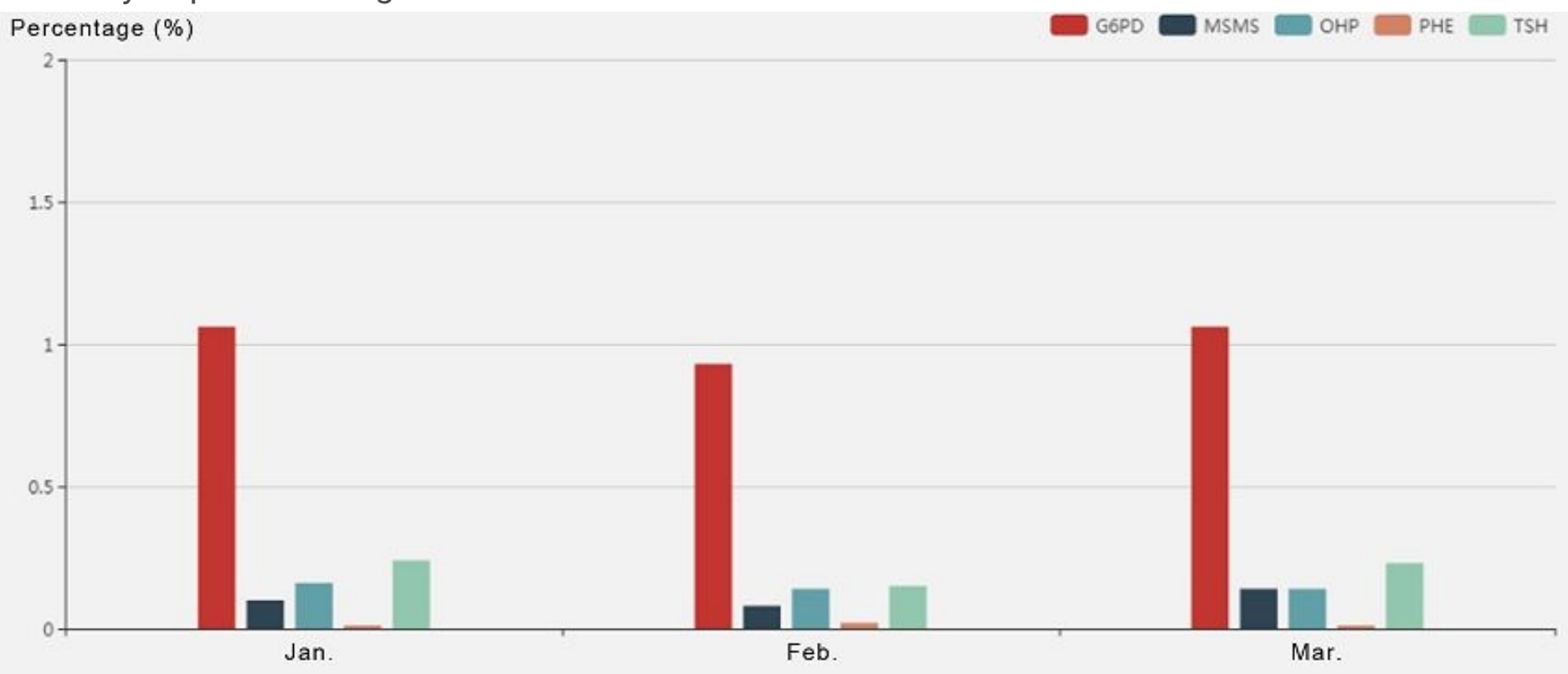

Figure 11

Screening positivity. 


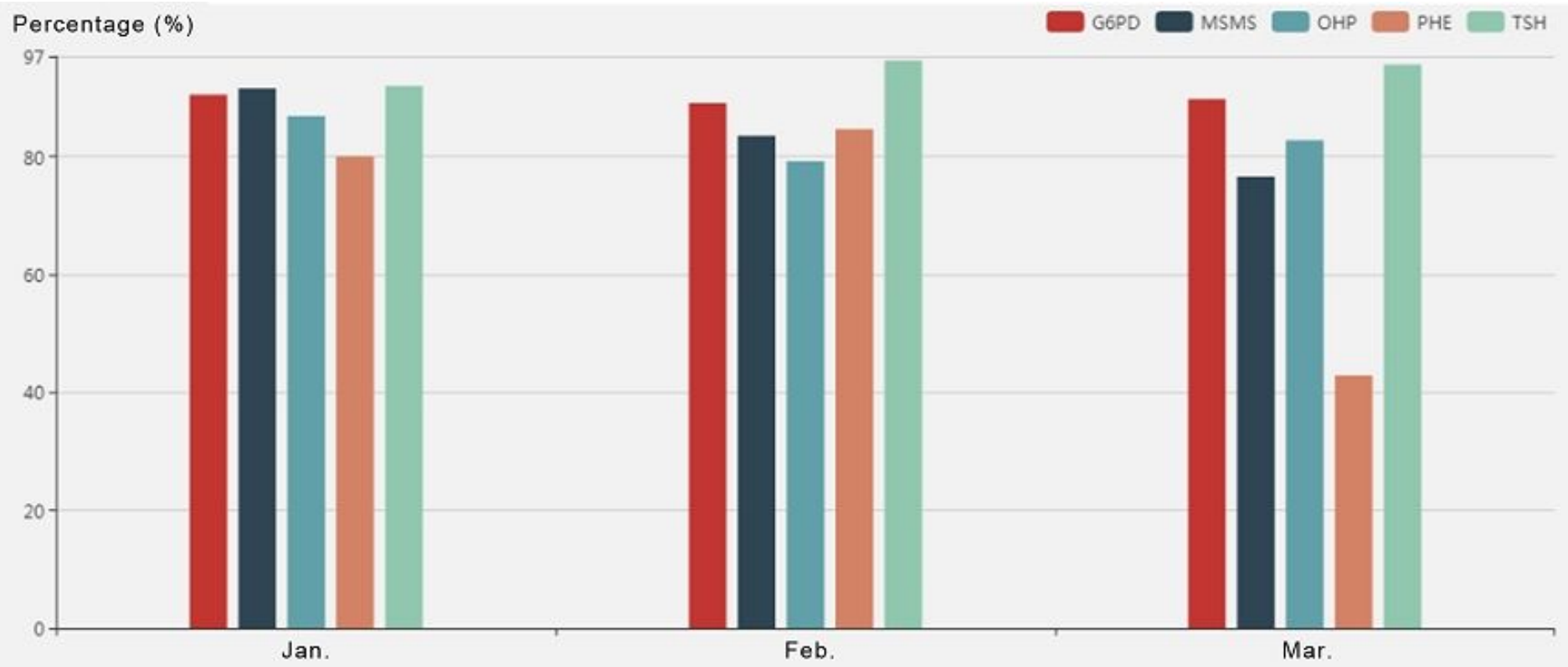

Figure 12

Recall rate of prescreening positivity.

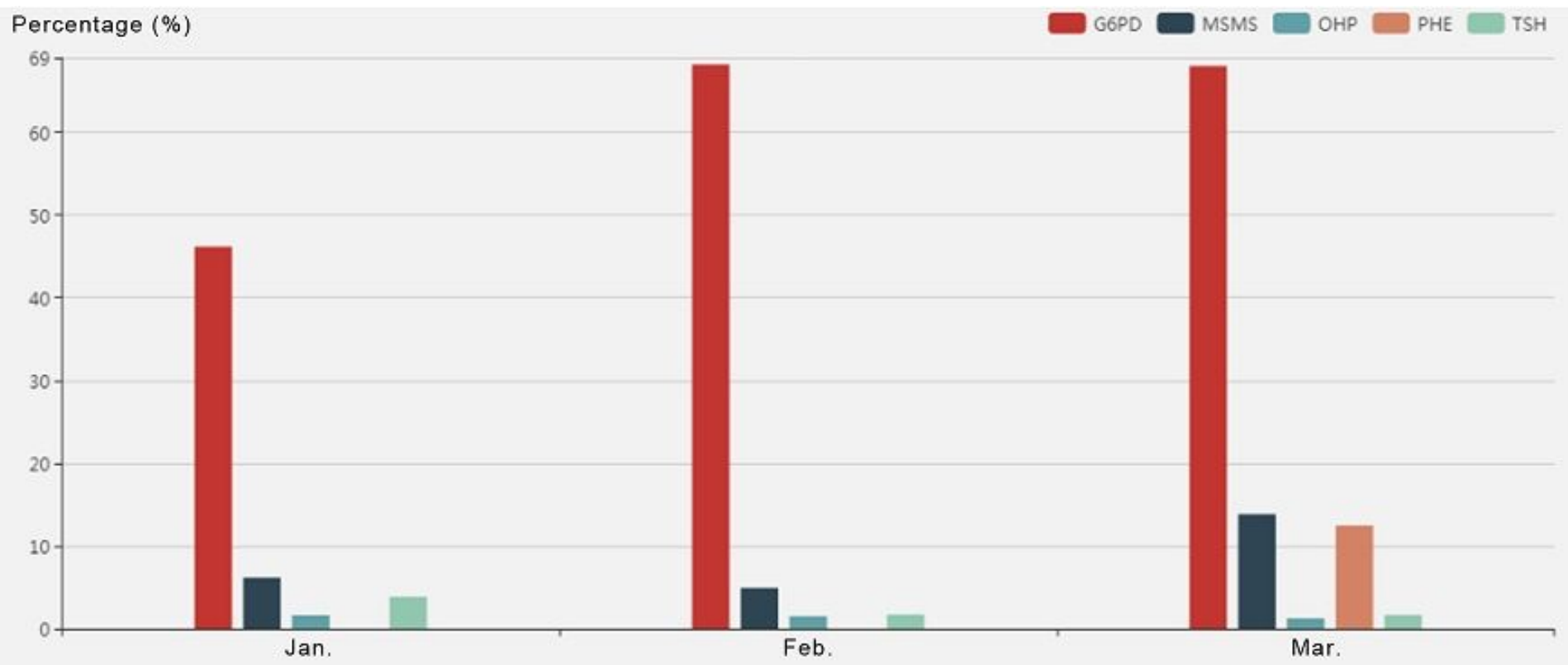

Figure 13

Recall positivity. 


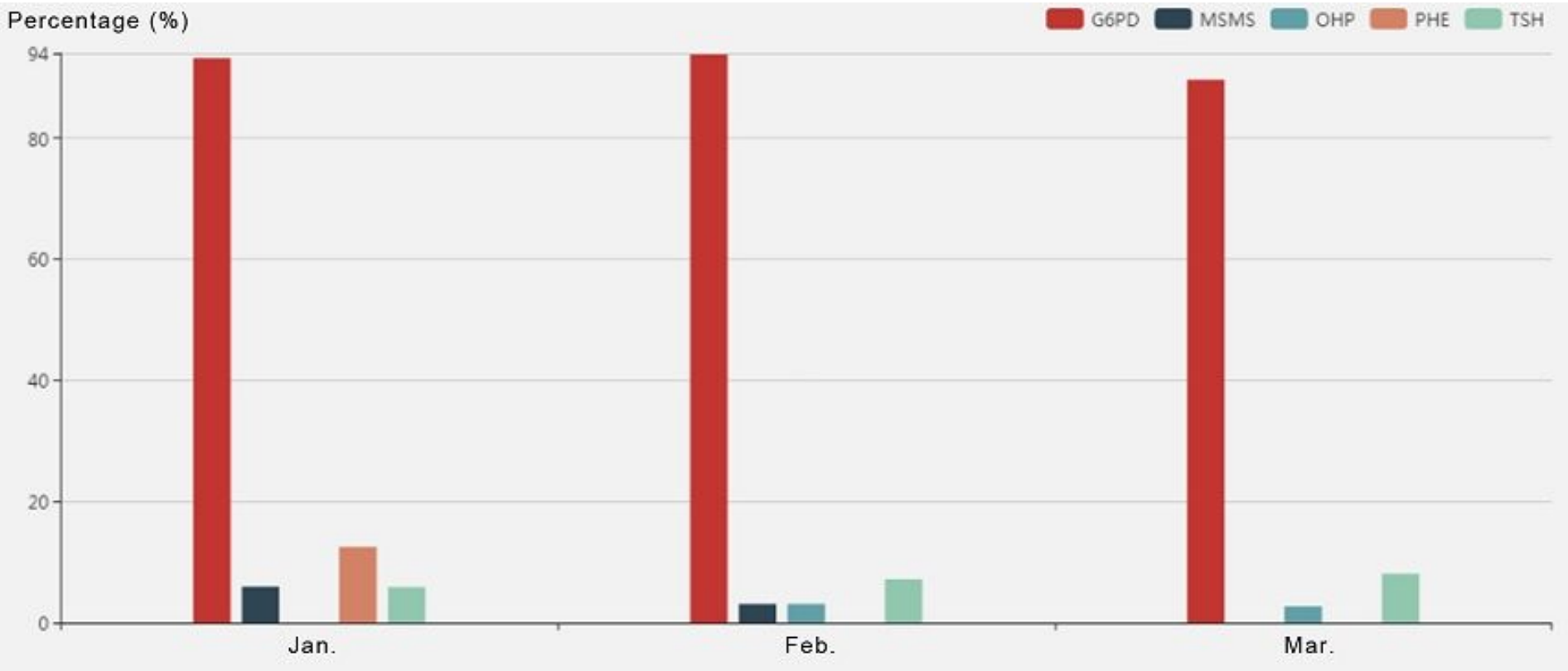

Figure 14

Positivity prediction.

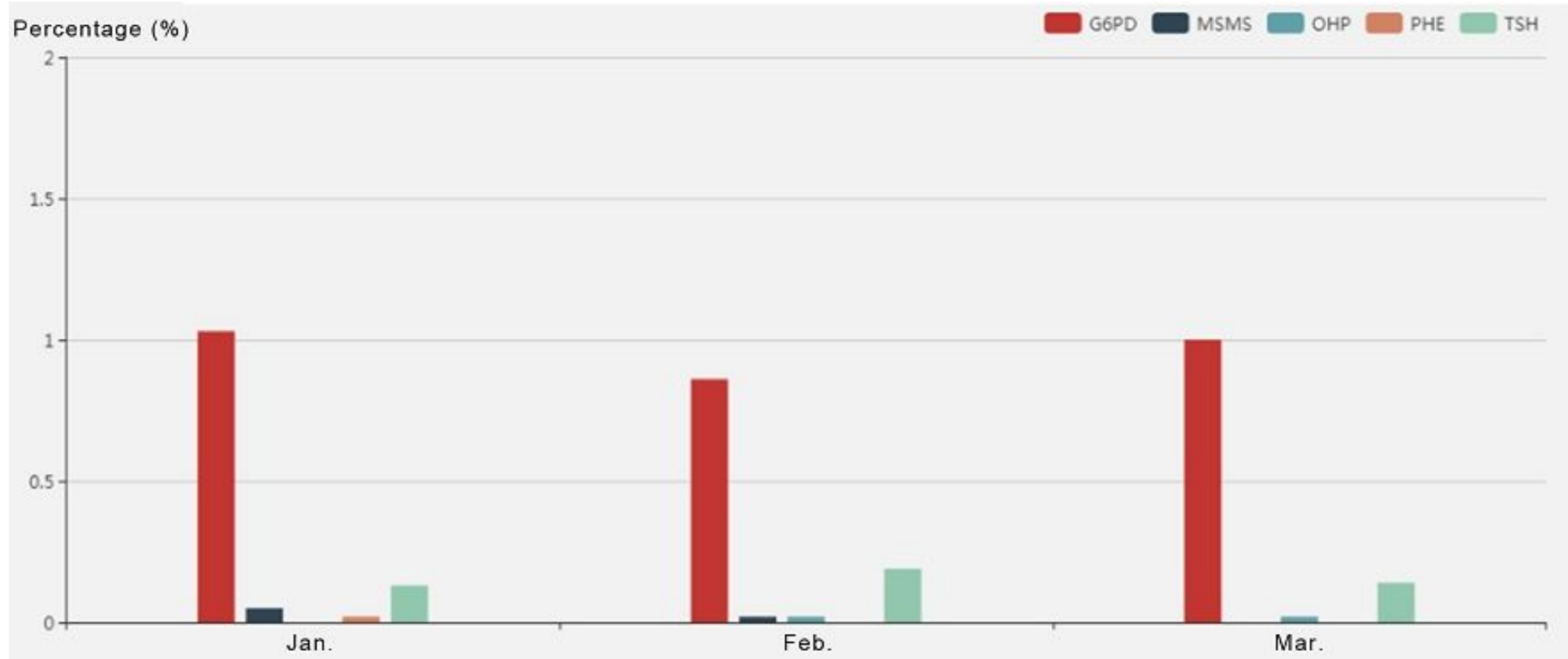

Figure 15

Lost of follow up. 